\title{
UTILIZANDO HERRAMIENTAS DIGITALES PARA ABRIR NUEVAS PERSPECTIVAS SOBRE LA HISTORIA DEL QUEHACER POLITIICO MAPUCHE
}

\section{JOANNA CROW* \& ALLISON RAMAY**}

En la época que siguió a la ocupación militar de La Araucanía (1861-1883) los intelectuales-activistas mapuche actuaron como intermediarios esenciales entre comunidades (un concepto entendido como algo más amplio que "reducción") y el Estado chileno. Educados en escuelas estatales y misioneras, utilizaron sus conocimientos tanto del mundo mapuche como del chileno para intentar mejorar, a través de sus escritos y quehacer político, la convivencia interétnica. Tres de los líderes que sobresalían en este escenario fueron Manuel Manquilef (1887-1950), Manuel Aburto Panguilef (18871952) y Venancio Coñuepán (1905-1968). Manquilef fue profesor, escritor e investigador, co-fundador y líder de la Sociedad Caupolicán, diputado (1926-1932) y gobernador de Lautaro (1936-1937). Aburto Panguilef fue intérprete para el Protectorado de indígenas en Valdivia, fundó la Sociedad Mapuche de Protección Mutua (1916) y una compañía de teatro y, desde 1921 en adelante, fue líder de la Federación Araucana que organizaba los Congresos Araucanos. Coñuepán fue gerente local de Ford Motors, fue líder de la Sociedad Caupolicán (1931), fundó la Corporación Araucana, Movimiento Indigenista de Chile (1938), fue dipu- tado (1945 y 1949) y Ministro de Tierras y Colonización y dirigió el Departamento de Asuntos Indígenas durante el gobierno de Carlos Ibáñez del Campo (1952-1958). Desde estos cargos, los tres produjeron múltiples textos (discursos, ensayos, cartas y, diario de vida, en el caso de Aburto Panguilef), también se escribió bastante sobre ellos y su trabajo (artículos de prensa, actas de las sesiones parlamentarias, historiografías y artículos académicos).

Existen numerosos e importantes estudios sobre estos documentos: por ejemplo, los trabajos de André Menard, José Ancán, Florencia Mallon, Jorge Pavez, Rolf Foester y Sonia Montecino ${ }^{1}$, los que analizan los discursos y estrategias políticas de estas tres figuras. Sabemos bastante sobre sus discursos y sus afiliaciones políticas pero parte de lo que aún se queda por explorar son los procesos específicos de cada uno para llegar a los diferentes cargos políticos que mantuvieron durante gran parte de sus vidas. ¿Con quiénes se relacionaban, cuándo, dónde y en qué contexto lo hacían? son las preguntas que nos llevaron a extraer datos de las publicaciones existentes (primarios y secundarios) para empezar a responder

\footnotetext{
* PhD. Senior Lecturer University of Bristol, Bristol, Reino Unido. Correo-e: jo.crow@bristol.ac.uk

** PhD. Profesora asistente de la Pontificia Universidad Católica de Chile, Facultad de Letras, Santiago, Chile. Correo-e: aramaya@uc.cl
} 
a estas interrogantes, y con la esperanza de entenderlos mejor a ellos como actores individuales y únicos y, a la misma vez, como parte de un colectivo dinámico y más grande. En esta misma línea, Nicholas Christakis y James Fowler, autores del premiado libro Connected nos instan a indagar sobre la relación entre las partes de un sistema y su totalidad, enfatizando que, en el siglo XXI en particular, hay un interés dentro de muchas disciplinas de hacer este esfuerzo; se ve este interés en los estudios sobre las neuronas en el cerebro, las macromoléculas en las células, y las personas en una red (Christakis \& Fowler, 2011).

A través del caso específico del proyecto "Historias Interconectadas: Redes de los intelectuales-activistas mapuche de principio de siglo XX" ("Historias Interconectadas") ${ }^{2}$ y de la página web que resultó de su primera fase ${ }^{3}$, quisiéramos analizar las ventajas como también las limitaciones de utilizar herramientas digitales para representar, a través de visualizaciones, datos sobre los mundos sociales de Manquilef, Aburto Panguilef y Coñuepán. Pretendemos delinear algunos de los debates relacionados con el uso de herramientas digitales en cuanto a preguntas de investigación que vienen desde las humanidades (las Humanidades Digitales o HD) para situar el proyecto y para explicitar algunos de los desafíos implicados en proyectos de este tipo.

Para empezar, reconocemos algunas de las fortalezas de una mirada que contempla los individuos históricos como parte de un sistema de redes más amplio. En su trabajo sobre el activista colombiano Juan Gregorio Palechor (1923-1992) Myriam Jimeno comenta (citando al antropólogo estadounidense Richard Werbner) que "muchos autores de historias de vida tienden a producir una especie de literatura confesional centrada en él/la protagonista como antihéroe, fugitivo u 'otro externo'” (2014, p. 22). Según Jimeno, Werbner pide "un nuevo tipo de biografía que ilustraría cómo las vidas de múltiples personas se entrelazan y propone una forma social o biografía que tomaría en cuenta los contextos y procesos sociales y establecer un diálogo entre varias personas dentro de una narrativa o narrativas" (2014, p. 22). Si bien la biografía se mantiene como un género interesante para los lectores del siglo XXI, también interesa la inclusión de distintas perspectivas sobre una misma vida; de esta manera nos acercamos más a una narrativa o mirada post-estructuralista y post-colonialista que considera varias "entradas" hacia la realidad y que permite la reconstrucción de un complejo sistema de relaciones (en vez de un "grand narrative" que surge desde una perspectiva única, hegemónica y patriarcal). Como se verá, utilizando herramientas que permiten la creación de un registro de nombres y sus posibles relaciones crea, desde luego, la posibilidad de un enfoque doble, tanto hacia el individuo como hacia una red dentro de la cual el individuo es solo un nodo.

\section{¿Por qué lo digital?}

Nuestro proyecto consideraba como elemento fundamental el uso de la tecnología tanto para la colaboración misma como para la realización y difusión del estudio. En sí, la colaboración vía Internet suele ser uno de los elementos básicos de cualquier proyecto en HD (Humanidades Digitales), pero los otros elementos que las constituyen son más difíciles de identificar. En la literatura existente es posible observar cierta reti- 
cencia a adherirse a una definición estricta sobre el significado de HD; algunos lo consideran un "campo" divergente de las humanidades y otros, un medio o método muchas veces entrelazado con las humanidades (Svensson, 2010). En general, sabemos que las "humanidades digitales" emplean una combinación de preguntas y acercamientos humanistas junto con herramientas digitales, lo que favorece la colaboración entre personas de distintas disciplinas y en diferentes partes del mundo. Como bien dice Lisa Spiro (2012):

en vez de intentar definir las humanidades digitales, debemos entenderlas como una comunidad que se reúne alrededor de valores comunes tales como la apertura y la colaboración...Cómo funcionan las humanidades digitales -de manera transparente, colaborativa, a través de redes en línea- las distingue. (p. 2) ${ }^{4}$

Nuestra propia metodología entendía este elemento como esencial ya que la colaboración misma nos potenciaba; cada una desde distintas disciplinas y con distintos accesos a documentos (originales y teóricos), y trabajábamos en base a la misma pregunta: ¿con quiénes conversaban, potencialmente, Manquilef, Aburto Panguilef y Coñuepán?

La definición que propone Tara McPherson de "multimodal humanities" coincide con algunos aspectos de nuestro trabajo, en el que la tecnología es más un medio, para proyectar visualmente información desde una base de datos, que un fin. Patrik Svensson (2010) habla sobre el trabajo de McPherson diciendo:

Esto no privilegia ni apunta especialmente a lo digital como un objeto de análisis, lo que puede ser en parte resultado de un foco más general en cuanto a la escritura multimodal en las humanidades en su totalidad, y se puede decir que es lo digital como medio de expresión la tendencia más común de las humanidades multimodales. $\left(\right.$ p.14) ${ }^{5}$
Tal como mencionan McPherson y Svensson, encontramos no un nuevo objeto de análisis en las visualizaciones, sino más bien un medio para cambiar la expresión y representación de los datos encontrados.

Este foco en la tecnología como medio y no un fin no es siempre la norma entre los investigadores y docentes de las humanidades digitales. Por ejemplo, David Golumbia (2014) dice: "En HD se sugiere a menudo que la construcción de herramientas digitales por si solo debe calificarse como un reemplazo de la escritura académica, una afirmación algo extraña y combativa en los departamentos de inglés (p. 160)"6. En nuestro caso, como se verá abajo, las herramientas utilizadas nos proveen con una mirada distinta de los archivos y los documentos escritos/publicados; una mirada de datos que provienen de un conjunto de textos/representaciones y que permiten ver las posibles interconexiones entre personas que movían y actuaban en el mismo espacio que Manquilef, Aburto Panguilef y Coñuepán.

\section{El caso de "Historias Interconectadas": EI corpus y el uso de herramientas digitales}

Durante el proyecto "Historias Interconectadas", que duró un año y medio, consultamos los archivos de la Biblioteca del Congreso Nacional y el Archivo Rodolfo Lenz en la UMCE e intercambiamos, de manera virtual, documentos primarios y secundarios como, por ejemplo, los Comentarios de Manquilef, el diario de vida de Aburto Panguilef (recién editado y publicado por Andrés Menard), las labores parlamentarias de Manquilef y Coñuepán, y la prensa regional y nacional de la época. De estos y muchos otros textos extrajimos la información sobre las perso- 
nas con quienes habían potencialmente interactuado los tres intelectuales. Incluimos distintos tipos de interacciones o encuentros registrados en los documentos que consultamos: por ejemplo, asistencia a la misma escuela, participación en los mismos congresos, organización de reuniones políticas, y asistencia a celebraciones o actos públicos. Registramos esta información en una base de datos con distintas columnas: el nombre del individuo conocido o encontrado, su relación con el intelectual que estudiamos, el tipo de encuentro donde ambos estuvieron, el lugar específico del evento o encuentro, la ubicación del evento (ciudad), las coordenadas geográficas del lugar de encuentro, la fecha de comienzo y término, y las fuentes primarias y secundarias. En este momento tenemos 250 conexiones para Manuel Manquilef, 300 para Manuel Aburto Panguilef y 248 para Venancio Coñuepán. Así se ve un fragmento de la base de datos para Manuel Manquilef:

\begin{tabular}{|c|c|c|c|c|c|c|c|c|c|c|c|}
\hline & Nombre & Relacion & Evento & Lugar de encuer & Ubicación & Coordenadas & \begin{tabular}{|l|} 
Fecha Evento \\
\end{tabular} & Fecha inicio & Fecha término & Fuente primaria & Fuente sec \\
\hline Manquilef & Tomás Guevara & Profesor de castella & Educación secunda & Liceo de Temuco & Temuco & $-38.733,-72.600$ & $1900-1902$ & 1900 & 1902 & Comentarios, 1911 , p. 5 & \\
\hline Manquilef & Salvador Castanede & Visitador de escuela & Educación secunda & Liceo de Temuco & Temuco & $-38.733,-72.600$ & 1900-1902 & 1900 & 1902 & Comentarios, 1911, p. 5 & \\
\hline Manquilef & Eulogio Robles & Profesor & Educación secunda & Liceo de Temuco & Temuco & $-38.733,-72.600$ & 1900-1902 & 1900 & 1902 & & \\
\hline Manquilef & Ramon Lienan & Compañero de cole & Educación secundal & Liceo de Temuco & Temuco & $-38.733,-72.600$ & $1900-1902$ & 1900 & 1902 & & Pavez, 'Escr \\
\hline Manquilef & Kolikeo Kidel/ Quide & Compañero de cole & Educación secunda & Liceo de Temuco & Temuco & $-38.733,-72.600$ & 1900-1902 & 1900 & 1902 & & Pavez, 'Escr \\
\hline Manquilef & Jose Segundo Pain & Compañero de cole & Educación secunda & Liceo de Temuco & Temuco & $-38.733,-72.600$ & 1900-1902 & 1900 & 1902 & & Pavez, 'Escr \\
\hline Manquilef & Salvador Castaneda & Lo recluta & Formación de profes & Escuela Normal ded & Chillan & $-36.600,-72.117$ & $1902-1906$ & 1902 & 1906 & & \\
\hline Manquilef & Manuel J. Ortiz & Profesor de castella & Formación de profes & Escuela Normal de & Chillan & $-36.600,-72.117$ & $1902-1906$ & 1902 & 1906 & & \\
\hline & & & & & & & & & & & \\
\hline Manquilef & A.C. Saldana & Colega & Empleado como pro & Internado Araucan & Quepe & $-38.850,-72.583$ & 1908-1909 & 1908 & 1909 & Comentarios, 1911 , p. 8 & Menard and \\
\hline Manquilef & Carlos Sadlier & Fundador de la Misi & Empleado como pro & Internado Araucan & Quepe & $-38.850,-72.583$ & 1908-1909 & 1908 & 1909 & & \\
\hline Manquilef & Georgie Sadlier & Profesor de las niña & Empleado como pro & Internado Araucan & Quepe & $-38.850,-72.583$ & 1908-1909 & 1908 & 1909 & Photo catalogued by Pav & vez and Men \\
\hline Manquilef & W. Sudger & Profesor principal & Empleado como pro & Internado Araucan & Quepe & $-38.850,-72.583$ & 1908-1909 & 1908 & 1909 & & \\
\hline Manquilef & Tomás Guevara & Rector (1900-1913) & Empleado como bib & Liceo de Temuco & Temuco & -72.600 & 1906-1907 & 1906 & 1907 & & ncl: \\
\hline Manquilef & Francisco Melivilu & Alumno o colega & Empleado como bib & \begin{tabular}{|l|} 
Liceo de Temuco \\
\end{tabular} & Temuco & $-38.733,-72.600$ & 1906-1907 & 1906 & 1907 & & www.ben.cl \\
\hline Manquilef & Fernando Navarette & Colega & Empleado como bib & Liceo de Temuco & Temuco & $-38.733,-72.600$ & 1906-1907 & 1906 & 1907 & & www.ben.cl \\
\hline & & & & & & & & & & & \\
\hline Manquilef & Leoncio Rivera & Miembro & Fundación del Atene & o de Temuco & Temuco & $-38.733 .-72.600$ & $? / 10 / 1907$ & & & & \\
\hline
\end{tabular}

Figura 1. Resultado no publicado del proyecto "Historias interconectadas"

Toda la información en las columnas tiene una fuente específica y aunque no podemos estar seguras de que los sujetos mencionados interactuaran directamente (algunas veces, solo vemos que estaban en el mismo lugar en el mismo momento), hay varios nombres que aparecen varias veces, lo que indica que probablemente jugaban un papel importante en el mundo social de los sujetos que estudiamos.
Una base de datos como esta es sumamente útil porque permite registrar de manera organizada grandes cantidades de información, pero quizás con la desventaja de ser demasiado estática. Entonces, con la ayuda de softwares disponibles en la Web como Graphiz y Palladio ${ }^{7}$ podemos transformar las columnas y filas en mapas y diagramas que reflejen la información de otra manera, como los que aparecen abajo: 


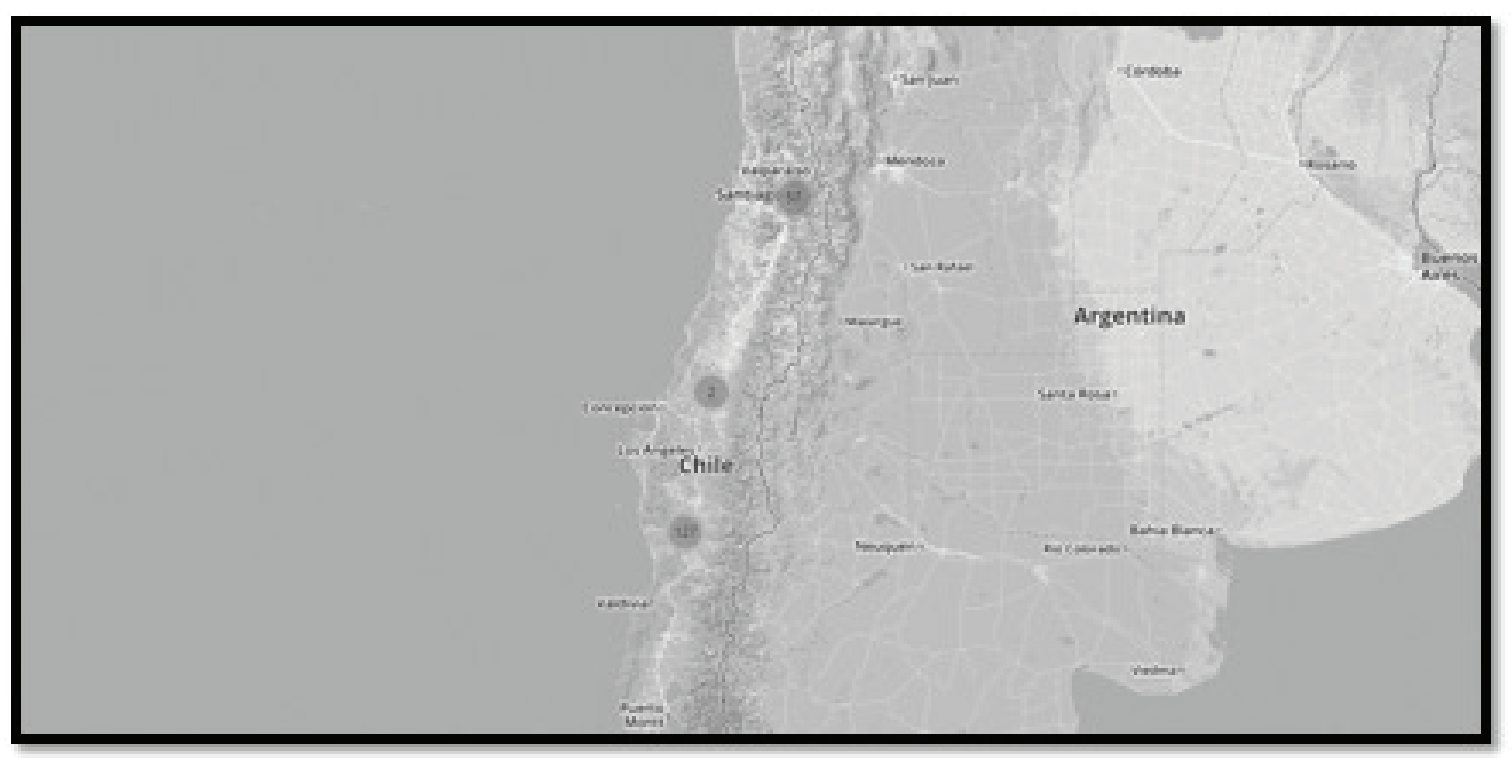

Figura 2. Resultado no publicado del proyecto "Historias interconectadas"

Los números que aparecen indican la cantidad de conexiones encontradas en cada área general del país (en el caso de Venancio Coñuepán se encuentra conexiones en México porque asistió al primer Congreso Interamericano Indigenista en Patzcuaro, México, en 1940). Si hacemos clic en uno de los círculos (Fig. 2), aparecen puntos que indican los eventos registrados y el nombre de las personas que asistieron. Como vemos en Figura 3 , según los archivos que consultamos y desde los cuales extrajimos datos, 15 eventos (los puntos sin un círculo blanco en medio) ocurrieron en Temuco en los cuales participó Manuel Manquilef. En total, en estos 15 eventos (que solo representan la punta del iceberg), Manquilef tuvo la posibilidad de conectarse, según las fuentes leídas, con 96 personas (los puntos con un círculo blanco en el centro). 


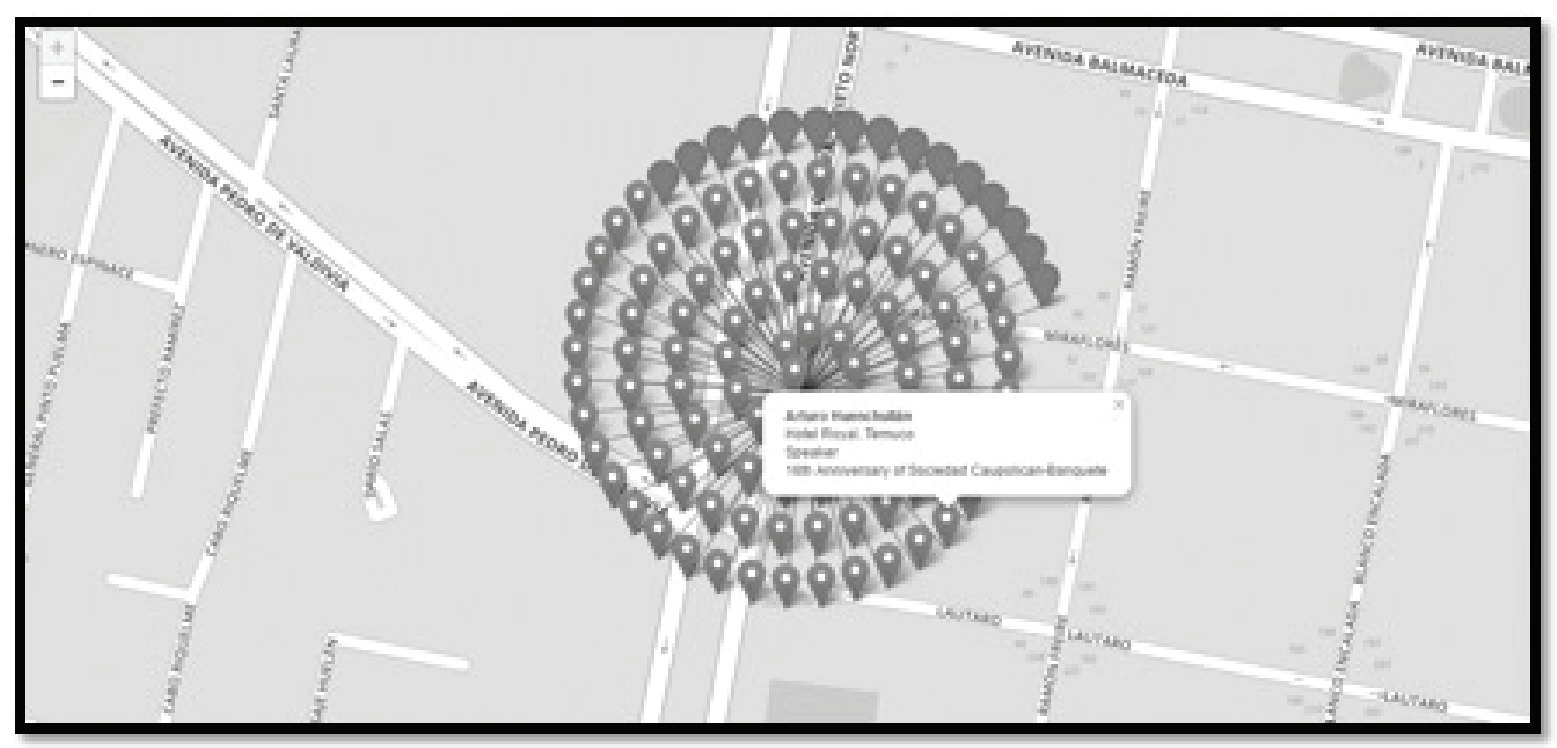

Figura 3. Resultado no publicado del proyecto "Historias interconectadas"

Otro tipo de visualización que hemos incor- nas con quien Manquilef tuvo mayor contacto porado a nuestra página son diagramas (Fig. (según los archivos consultados), solo tenemos 4), las que vinculan visualmente a las personas que buscar los "cuadrados" con más líneas y los encuentros. Para identificar a las perso- apuntando hacia ellos.

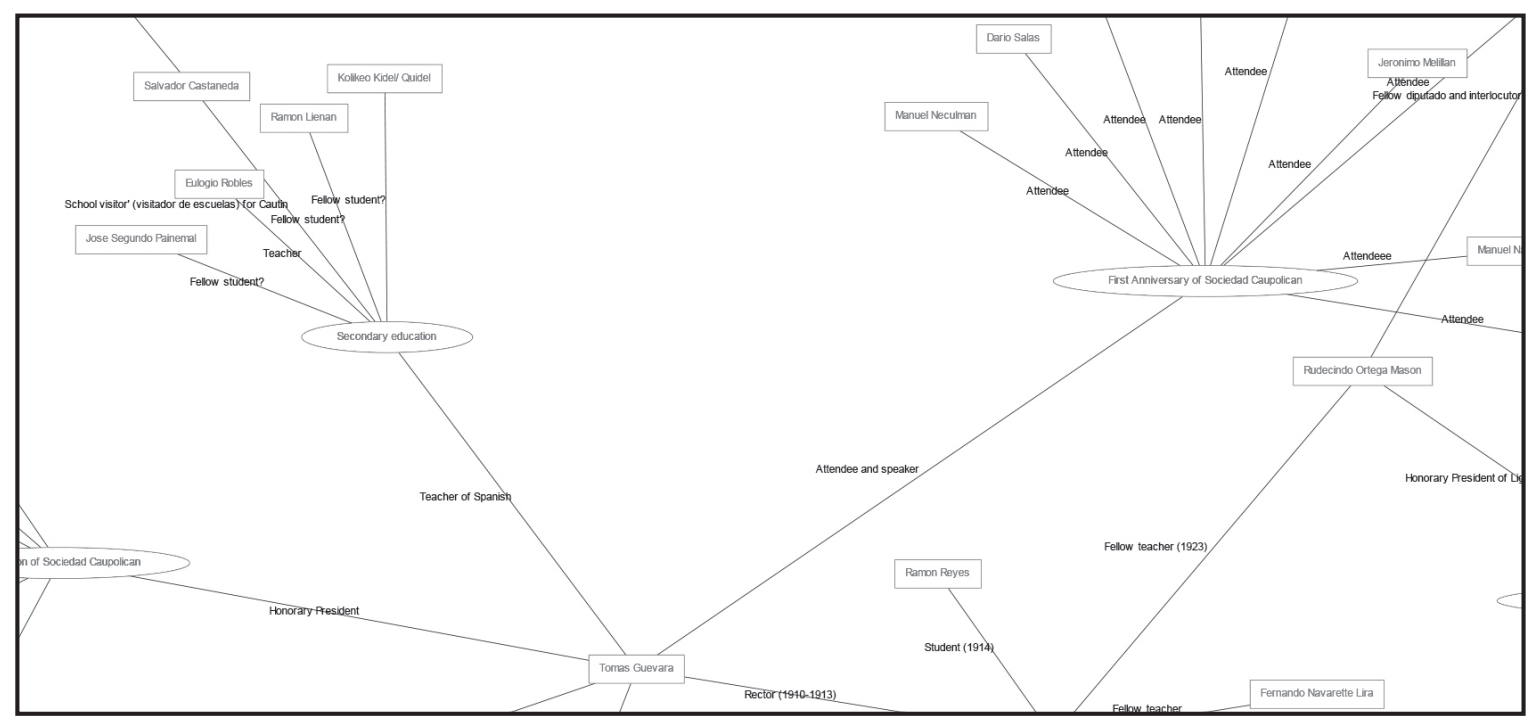

Figura 4. Resultado no publicado del proyecto "Historias interconectadas" 
Se encuentra la misma información que la de la base de datos pero desplegada de manera espacial. Si bien sería posible usar otras formas de visualización como, por ejemplo, una tabla de doble entrada que cuantifique las relaciones entre autores y eventos, el diagrama permite la identificación visual de las personas que aparecen más en los documentos, facilitando el análisis de la información. Vemos, por ejemplo, personas que estuvieron presentes en muchos de los mismos eventos a los que fue Manquilef (Tomas Guevara, Eulogio Robles y Carlos Sadlier son algunos de los nombres más sobresalientes). Aunque ya se ha investigado con bastante detalle la relación entre Manquilef y Tomás Guevara ${ }^{8}$, aún hay mucho por saber sobre su relación con otros sujetos como Sadlier y Robles y la influencia que pudieron haber tenido en el discurso y la trayectoria intelectual y política de Manquilef. En este sentido, la repetición de los nombres funciona como una invitación a indagar sobre la posible relación y el papel de cada uno de los sujetos en el movimiento mapuche del momento.

Una de las ventajas de visualizar los datos de esta forma es que podemos identificar fácilmente a las personas que participaban en los espacios sociales de los actores específicos que hemos estudiado; emergen sujetos que se encontraban en los mismos espacios que los sujetos más visibles pero que, muchas veces, no han recibido tanta atención dentro de las investigaciones actuales. Este es el caso de Rudecindo Ortega Mason, cuyo camino de vida profesional está entrelazado con el de Manquilef pero cuyo nombre no aparece en las publicaciones actuales sobre este. Ambos fueron profesores en el Liceo de Temuco y elegidos al mismo tiempo como diputados (Ortega del Partido Radical y Manquilef, del Partido Libe- ral) para Imperial, Temuco y Villarrica en 1925 y reelegidos en 1930. Ortega también fue, junto con Manquilef, co-fundador (y después presidente) de la fundación de la Liga de Estudiantes Pobres de Temuco. Aunque no existe registro conocido de sus conversaciones, sabemos que Manquilef y Ortega circulaban en las mismas esferas sociales, por lo que es posible suponer que se influían mutuamente. Este tipo de análisis nos invita, entonces, a considerar el significado de otras historias en relación con aquellas que ya conocemos, enriqueciendo e incentivando el estudio y entendimiento del activismo político mapuche durante las primeras décadas del siglo XX, a través de los interlocutores chilenos e interacciones entre mapuche y no-mapuche.

\section{Las ventajas de bases de datos y la visualización de documentos históricos}

En "Conjectures on World Literature”, el teórico cultural Franco Moretti (2000) define el concepto de "distant reading" así: "donde la distancia [...] es una condición del conocimiento: te permite enfocarte en las unidades que son más chicas o más grandes que el texto: dispositivos, temas y tropos, o géneros y sistemas" (p. 57) ${ }^{9}$. Uno de sus puntos centrales es que la tecnología abre la posibilidad de estudiar un sistema de relaciones; las relaciones entre los personajes de una novela, por ejemplo, en vez de los personajes por separado. En otro artículo, Moretti (2011) habla de las ventajas de convertir el tiempo en espacio o de ver en un solo pantallazo las conexiones e interrelaciones que existían en múltiples momentos históricos. "¿Qué sacamos al transformar el tiempo en espacio?", pregunta Moretti, y contesta: 
Primero que nada esto: cuando vemos una obra de teatro, estamos siempre en el presente: lo que está en el escenario, es; y luego desaparece. Aquí [en los mapas que visualizan todas las relaciones de un personaje en un drama o una novela], nunca nada desaparece (...) El pasado deviene en el pasado, sí, pero nunca desaparece de nuestra percepción de la trama". (p. 3-4) $)^{10}$

Al representar el tiempo no de manera unidireccional, los mapas tienen la capacidad de insistir en el aspecto penetrante del tiempo histórico en el tiempo del presente.

En nuestra experiencia, una mirada desde la distancia (lo que posibilita los diagramas) es más potente si va combinada con una mirada enfocada en la historia de ciertos individuos o ciertos lugares de encuentros. Por ejemplo, si tomamos en consideración que Manuel Manquilef conoce a Manuel J. Ortiz (otra figura poco estudiada hasta ahora) en la Escuela Normal de Chillán y que luego Manquilef dedica uno de sus "Comentarios" a Ortiz, entendemos que hay una relación intelectual que probablemente partió por un contacto interpersonal. Entonces, hay un diálogo por descubrir entre los diagramas y los sujetos representados. De acuerdo con Janicke, Franzini, Chemma y Scheuermann (2015), entonces, la lectura a la distancia no puede reemplazar "close reading". Lo que sí puede hacer es advertirnos de ciertos patrones. Como dice Janicke et. al:

una tarea importante para el desarrollo de visualizaciones es el proveer una visión amplia de los datos que subrayan patrones potencialmente interesantes. Enfocarse en estos patrones para luego explorarlos en detalle, constituye el puente entre la lectura a la distancia y de cerca. (n.p.) ${ }^{11}$
La base de datos nos permite registrar detalles que tal vez no aparezcan como significativos en el "close reading", pero que sí nos entregan información fascinante como "distanced reading". Por ejemplo, cuando nos enfrentamos con un diario de vida, como el diario de vida de Manuel Aburto Panguilef, tendemos a enfocarnos en lo que el diario nos cuenta sobre la persona que escribe (o quien entrega el contenido) del diario. En el prólogo del diario, escrito por André Menard (2013), el mismo que lo compiló, nos cuenta muchos detalles sobre el pensamiento de Aburto, enfocándose en la pregunta "¿por qué escribió tanto?" (p. XIX). Justamente, algo que incluye Aburto Panguilef en su diario son los nombres de cientos de personas con quienes se encontraba (por trabajo, ocio, etc.) y estos detalles nos permiten ver la "historia" de Aburto Panguilef desde un ángulo que des-enfatiza el papel del hombre "central" del relato. De hecho, el diagrama nos hace ver que una de las personas más mencionadas en el diario es Hipolito Mendez Ortiz, una persona no-mapuche que fue clave en los giros de la compañía de teatro de Aburto Panguilef. Además, se resalta de manera maravillosa las múltiples mujeres (sus nombres y los trabajos que realizaban) con quienes interactuaba Aburto Panguilef. En este sentido, los detalles encontrados en el diario permiten la construcción de una historiografía que, en contraste con historiografías tradicionales sobre "hombres importantes", nos exigen mirar a las personas que influyeron directamente en la visibilidad, el liderazgo e incluso en el "éxito" de individuos que tienden a ser los más vistos o considerados (tal como Aburto Panguilef).

Las visualizaciones, entonces, pueden ser un punto de entrada hacia la información para un público no familiarizado (o poco familiari- 
zado) con la historia del movimiento mapuche y de los activistas-líderes de principios del siglo XX, sobre todo porque parecen ser más impactantes que una base de datos. Nuestro interés primordial es invitar a los lectores a entender la construcción de estas visualizaciones de modo que sirvan como un espacio para indagar sobre ciertos encuentros, relaciones y lugares de encuentro, a la vez que participar en la expansión de nuestro conocimiento sobre el activismo político mapuche. Para entender mejor los eventos o a las personas, el usuario puede volver a la base de datos y así ver las fuentes utilizadas. En algunos casos tenemos disponibles en la página web las mismas fuentes (por ejemplo, los artículos de prensa) consultadas.

De alguna manera, lo que estamos documentando es cómo se ha construido o cómo se ha narrado la historia de los intelectuales y políticos mapuche de la primera mitad del siglo XX. Estamos documentando de manera abierta y transparente no solo nuestro proceso de investigación, sino también el proceso más generalizado de la investigación historiográfica en Chile y la producción de documentos escritos, en algunos casos por los mismos intelectuales indígenas y en otros, por otros que escribían sobre ellos. La base de datos es, entonces, un archivo de archivos escritos (que hasta ahora no incluye textos orales).

Por otra parte, el proyecto busca responder a las observaciones que otros estudiosos han hecho sobre la historia mapuche en Chile. Por ejemplo José Ancán (2014) ha subrayado que:

conocer más de la figura y obras de Manuel Mañkelef implica hoy un gesto cargado de urgencias. Más todavía cuando es la propia situación política de La Araucanía interétnica, y del país actual, lo que da sentido y razón a estos probables análisis. (p. 13)
Nosotras nos identificamos con esta urgencia. Leyendo lo que se ha escrito al respecto, observamos vacíos en su historia y la de otros activistas-intelectuales: de cómo llegaron a ser políticos (y asociados a ciertos partidos nacionales) ${ }^{12}$, cómo iban cambiando sus ideas y dónde viajaban durante sus carreras, entre muchas otras interrogantes. Conocer sus círculos sociales podría ayudarnos a entender más sobre su contexto y compartir esta información a través de una página web con imágenes visuales también podría ser una forma de llegar a un público más amplio para el cual el trabajo académico tradicional no está dirigido.

En esta misma línea, Pablo Marimán observa que existe una "fosilización de la palabra" en Chile, lo que quiere decir que la historia sobre los mapuche deja de fluir y renovarse (Pavez, 2003) ${ }^{13}$. Pensamos que la tecnología digital, en general -y este tipo de uso en particular-, puede contribuir a promover un entendimiento de la historia como un ente vivo (nuestras lecturas de ella cambian según el paradigma en el que vivimos, y dependiendo de las fuentes que leemos). Las visualizaciones vayan renovándose mientras insertamos más información sobre las vidas y las trayectorias políticas e intelectuales de Manquilef, Aburto Panguilef y Coñuepán.

\section{Desafíos dentro de HD}

Mientras nosotras y las personas que hemos consultado en el último año y medio hemos apreciado la contribución de los resultados de nuestro proyecto, también ha habido varias dudas sobre las metodologías para proyectos en las humanidades que incluyen el uso de herramien- 
tas digitales. Por una parte, vemos el problema generalizado de que el interés paradigmático de HD parece estar mucho más presente, al menos de manera formal, en el mundo anglosajón (que en el mundo hispano), donde hay instituciones que incentivan propuestas específicamente de "digital humanities"14. Hemos visto en Chile esfuerzos por indagar y reflexionar sobre cómo abordar la relación entre la tecnología y las humanidades (para la investigación y docencia), pero hasta ahora no hemos encontrado ninguna entidad que entregue fondos específicamente para proyectos en esta área.

En las palabras de Gayatri Spivak, y recordándose de las palabras de Raymond Williams, existe un problema de la "global digital divide" (Paulson, 2016) (división global y digital) en el que los accesos a las herramientas digitales y al internet mismo no son uniformes. Brown y Nicholas (2012) también sostienen esta idea en su artículo sobre la protección de la propiedad cultura indígena: "una división digital aun afecta la accesibilidad en relación a dispositivos y sistemas para hacer redes para los que son marginalizados en cualquier sistema político o económico" (pp. 308-309) ${ }^{15}$. En este sentido, el concepto de la democratización del conocimiento a través del Internet, sigue siendo problemático.

Desde el mundo anglosajón, hay proyectos de humanidades digitales que han nutrido nuestro propio proceso. Uno de estos es "Mapping the Republic of Letters"16, un recurso imprescindible para entender las redes de los intelectuales europeos durante los siglos XVII y XVIII. La página web ofrece estudios de casos que demuestran cómo viajaron o circularon las ideas de la llustración a través del intercambio epistolar. Los investigadores registran datos sobre las cartas como, por ejemplo, la fecha, el origen de cada carta, el género del receptor y emisor, etc., los que ayudan a dar respuesta a preguntas concretas sobre el movimiento de cada intelectual y el patrón de sus redes. Provee estadísticas y documentos originales de una manera visualmente atractiva (por ejemplo, incluye gráficos que reflejan la cantidad y el camino de las cartas envidadas por Galileo durante varias décadas). Con la excepción del proyecto sobre D'Alembert, el enfoque está centrado en los datos de un grupo de cartas, pero no en la información en ellas contenida. En cambio, en nuestro proyecto, visualizamos datos específicos extraídos de documentos históricos para facilitar un análisis de las relaciones de los intelectuales y los hechos históricos en que participaron. Adicionalmente nuestra idea es fomentar la colaboración de otras personas para completar la información recolectada, lo que permitirá que los usuarios incorporen datos desde sus propias lecturas y, de esta manera se podrá visualizar la participación, aun de manera indirecta, de miles de sujetos con el movimiento mapuche de principios del siglo XX. Para que esto sea posible, el próximo desafío será incorporar a la página web la capacidad de colaboración virtual de diferentes personas para generar y editar contenido.

Tal vez el desafío más evidente, en términos prácticos, de convertir datos en visualizaciones es la necesidad de invertir tiempo y recursos (humanos) en conocer las herramientas disponibles y aprender a usarlas. Aunque existen investigadores en humanidades que tienen conocimientos de programación, no parece ser la norma ${ }^{17}$. En nuestro caso, los fondos que conseguimos eran para organizar talleres y facilitar las conversaciones entre nuestras instituciones universitarias, no para la formación en TI o para contratar de manera formal 
a algún experto en TI. Para crear los diagramas y mapas, dependíamos de un profesional de tecnologías de la información (TI) que no trabajaba exclusivamente en nuestro proyecto. Gracias a la buena disposición de esta persona pudimos cumplir con lo que nos propusimos, pero también nos dimos cuenta de que muchas de las ideas que tuvimos durante el proyecto para hacerlo más atractivo e interactivo no eran viables sin un fondo más sustancial.

Más allá de nuestros propios intereses investigativos, una de las grandes motivaciones de hacer este proyecto - en una futura fase - es la posibilidad de proveer un espacio para que un público amplio pueda contemplar e investigar sobre las inter-relaciones e inter-conexiones entre personas (mapuche y no-mapuche) que participaban (directa o indirectamente) en los albores del movimiento mapuche. Tal como se encuentran los diagramas, las identidades de las personas presentes no están asociadas a ningún tipo de categoría étnica. Sabemos que hay una mezcla de sujetos que se auto-identificaban como mapuche y otros que no, y los diagramas son una representación que no distingue entre diferentes identidades, dejando incompleto y ambiguo este elemento de cómo pensamos la historia, permitiendo que el/la lector/a considere además de identificarse como mapuche, podemos entender y apreciar a estos actores a través de otras categorías (por los cargos que asumieron, el trabajo que hicieron) y por sus relaciones con y en relación con otros.

En este sentido, el acercamiento a través de la recolección de datos asume que Manquilef, Aburto Panguilef y Coñuepán fueron agentes importantes en su momento, cuyo trabajo tuvo consecuencias reales en las sociedades en las que vivían. No es una página que busca re-crear oposiciones binarias entre identidades, sino que busca dejar a la vista los posibles encuentros e interconexiones y dar mayor luz al trabajo realizado por estas tres figuras con la posibilidad de investigar (indagar en las mismas fuentes registradas), desde diferentes ángulos disciplinares: lingüística, literatura, historia, sociología, etc.

También es cierto que los archivos que consultamos forman parte de la historia de sujetos que vivieron y lucharon en contra de actitudes y legislaciones colonizantes; son sujetos que intentaron ganar espacios dentro de instituciones (y en relación a las personas ahí encontradas) que muchas veces no querían escuchar ni aprender de ellos. Son figuras con historias de subalternidad pero en la página que hemos construido, esa no es la lectura principal o única que se puede hacer de ellos.

En relación a la proyección y manipulación del conocimiento indígena por herramientas digitales, Radhika Gajjala (2013), Profesora de Comunicación y Medios Virtuales, advierte:

una y otra vez, afirmamos nuestra autoridad para desci-
frar los conocimientos y alfabetizaciones de Otros para
hacerlos disponibles para la apropiación y consumo en
lo Global. Se trata de cómo los congelamos, momifica-
mos y museo-mizamos (...). (p. 23)

En la página que ofrecemos, los textos incluidos son representaciones y auto-representaciones (con la excepción de las sesiones parlamentarias y el diario de vida de Aburto Panguilef) que se presentan en formatos/géneros occidentales. Dado que estamos seleccionando información desde representaciones, surge la noción de que las bases de datos y las visualizaciones son una especie de meta-archivo; un archivo compuesto por diferentes tipos de archi- 
vos (la mayoría estatales y algunos más bien mapuche, como el diario de Aburto Panguilef).

En definitiva, es un meta-archivo que intenta contestar y desafiar el concepto tradicional del archivo como repositorio privado y poco-accesible, tal como vemos en tantos otros esfuerzos en Chile $^{18}$. Al mismo tiempo, estamos conscientes del problema al cual se refirió Gayatri Spivak (2016) cuando estuvo en Chile (agosto, 2016) en cuanto al uso de lo digital: "Los proyectos digitales", dijo, hablando en la Universidad de Chile, "sirven principalmente para dar a conocer a los investigadores que los crean". Hemos intentado disminuir la presencia de nuestros nombres (solo aparecen en una sección) y hemos dado más prioridad a los textos y las visualizaciones que a nuestros propios análisis. Aunque hemos delineado el criterio y las categorías para organizar los datos, y hemos elegido las categorías que se visualizan en los diagramas y mapas, las fuentes y las historias que cuentan los archivos son el enfoque, por encima de nuestras historias.

Otra preocupación que expresa Spivak es que lo digital puede estar relacionado con la trivialización de las humanidades en el siglo $\mathrm{XXI}$; el ambiente actual en el que buscamos acelerar los procesos de aprendizaje (a través de la digitalización, por ejemplo) e intentamos convertirnos en seres "útiles" en vez de seres pensantes. A través de un proceso que involucra reflexión y atención a nuestras identidades locales (en relación y comunicación con lo global), Spivak insinúa que puede haber buenos usos de la tecnología ${ }^{19}$. Este concepto sí parece relacionarse con los fines del proyecto que aquí hemos descrito ya que para los usuarios que viven en Chile, ofrece elementos de la historia nacional que no han sido incluidos de manera sistemática en los textos escolares; la página es una invitación para nuevos acercamientos a las fuentes originales por parte de otros investigadores.

\section{Conclusiones}

Si somos capaces de hallar una manera de superar las barreras de las humanidades digitales (acceso desigual al Internet y a las personas con conocimiento para crear programas digitales, como también la posible trivialización de las humanidades como efecto de lo digital), podrían constituir un espacio en el que alumnos e investigadores encuentren formas de hacerse sus propias preguntas investigativas y pensarlas desde otras perspectivas. En nuestro caso, lo más gratificante ha sido el proceso de ver una historia descentralizada; una que parte por las vidas de Manquilef, Aburto Panguilef y Coñuepán y que se desplaza hacia sus redes. Hemos aprendido más sobre los tres intelectuales-activistas que constituyen el enfoque principal del estudio, pero también vimos la emergencia de otros sujetos sobre los cuales sabíamos muy poco o nada. Pudimos reafirmar la noción de que los líderes nunca actúan solos y que en realidad estos tres sujetos son tres nodos en una red de infinitas conexiones.

Estamos convencidas de que nuestro proyecto entrega una mirada distinta y productiva sobre la construcción de la historia; las personas que quieren saber más tienen la posibilidad de indagar a través de los mismos documentos y de llenar algunos de los vacíos que se encuentran. El éxito que percibimos del proyecto también tiene que ver con el hecho de que las herramientas que elegimos responden al tenor de nuestras preguntas y perplejidades, 
y no al revés. Partimos desde nuestras inquietudes específicas como investigadoras de las humanidades, y en este sentido, las herramientas digitales han sido un medio, no un fin. Con más instituciones en Chile entregando fondos para el trabajo colaborativo e interdisciplinarios para que las personas con conocimiento especializado en las humanidades, por un lado, e ingeniería o ciencias computacionales, por otro, trabajen en conversación, cuando sea posible, junto con los descendientes de los sujetos históricos, pensamos que las humanidades digitales ofrecen un gran espacio de reconocimiento y respeto hacia las múltiples historias y perspectivas contenidas dentro del concepto de historia nacional.

\section{Notas}

\footnotetext{
${ }^{1}$ Ancán, José (2014). De küme molfüñche a 'civilizados a medias': liderazgos étnicos e intelectuales mapuche en la Araucanía fronteriza (1883-1930), Polis, Revista de la Universidad Bolivariana, 13(38), pp. 19-44; Ancán, José (2008). Venancio Coñuepán (Santiago: Ediciones Usach); Foerster, Rolf y Sonia Montecino (1988). Organizaciones, líderes y contiendas mapuches (1900-1970) (Santiago: CEM); Mallon, Florencia (2009). El siglo XX mapuche: Esferas públicas, sueños de autodeterminación y articulaciones internacionales. En Christian Martínez y Marco Estrada (editores). Las disputas por la etnicidad en América Latina: Movilizaciones indígenas en Chiapas y Araucanía (Santiago: Catalonia), pp. 155-190; Mallon, Florencia (2010). La 'Doble columna' y la 'Doble conciencia' en la obra de Manuel Manquilef, Revista Chilena de Antropología 21, pp. 59-80; Marimán, Pablo (1999). Coñuepán en el parlamento de 1947: argumentos y propuestas de la Corporación Araucana, Liwen 5, pp. 157-175; Menard, André (2006). Emergencia de la tercera columna en La Faz social de Manuel Manquilef, Anales de Desclasificación, I(2), pp. 927-948; Menard, André y Jorge Pavez (2007). Mapuche y Anglicanos: Vestigios fotográficos de la Misión Araucana de Kepe, 1896-1908 (Santiago: Ocho Libros); Menard, André y Jorge Pavez (2005). El Congreso Araucano, Raza y Escritura en la Política Mapuche. Política 44, pp. 211-232.

${ }^{2}$ El proyecto recibió financiamiento de dos fondos cuyo objetivo principal era la colaboración entre investigadores e instituciones académicas de Chile y el Reino Unido: del British Academy ganamos el concurso del "International Partnership and Mobility Scheme" y de Conicyt, el de Newton-Picarte: "Redes internacionales".

${ }^{3} \mathrm{http}: / /$ www.bristol.ac.uk/arts/research/historias-interconectadas/

${ }^{4}$ En texto original: "....rather than try to define digital humanities, we should understand it as "a community that comes together around common values such as openness and collaboration... How the digital humanities community operates-transparently, collaboratively, through online networks-distinguishes it."

${ }^{5}$ En texto original: "It does not privilege nor explicitly single out the digital as an object of analysis, which partly may be a result of
}

a more general focus on multimodal writing in the humanities at large, and arguably the most prominent mode of engagement for the multimodal humanities is the digital as an expressive medium."

${ }^{6}$ En el texto original: "DH repeatedly suggests that the building of computational tools in itself should qualify as a replacement for scholarly writing, a particular odd and combative claim in English departments..."

${ }^{7}$ http://hdlab.stanford.edu/projects/palladio/

${ }^{8}$ Véase, por ejemplo, Jorge Pavez (2003): 'Mapuche ñi nütram chilkatun / Escribir la historia mapuche', Revista de Historia Indígena 7, pp. 7-53.

${ }^{9}$ En el texto original: "Where distance....is a condition of knowledge: it allows you to focus on units that are much smaller or much larger than the text: devices, themes, tropes - or genres and systems."

${ }^{10}$ En el texto original: "What do we gain by turning time into space? First of all this: When we watch a play we are always in the present: what is on stage is, and then disappears. Here, nothing ever disappears. (...) The past becomes past, yes, but it never disappears from our perception of the plot."

${ }^{11}$ En el texto original: "an important task for the development of visualizations is to provide an overview of the data that highlights potentially interesting patterns. A drill down on these patterns for further exploration is the bridge between distant and close reading."

${ }^{12}$ En el caso de Manquilef, es el Partido Liberal Democrático.

${ }^{13}$ Véase también Pablo Marimán (1995): Demanda por educación en el movimiento mapuche en Chile: 1910-1995. Temuko: Relmu.

${ }^{14}$ En España hemos tenido contacto con tres instituciones que son referentes para las HD para el mundo en español: 1. Medialab UGR en Granada, España (Esteban Romero) y 2. El primer Laboratorio de Innovación en Humanidades Digitales en español www.linhd.uned. es (Elena González-Blanco) y 3. La Asociación de Humanidades Digitales Hispánicas: http://www.humanidadesdigitales.org/ (Elena González-Blanco)

${ }^{15}$ En el texto original: "a digital divide still affects accessibility to both devices and systems of networking for those marginalized in 
any economic or political system."

${ }^{16}$ http://republicofletters.stanford.edu/index.html

${ }^{17}$ Ryan Shaw reflexiona sobre esto en la charla que realizó el día 25 de agosto, 2014 en la Pontificia Universidad Católica de Chile, Santiago, Chile.( https://www.youtube.com/watch?v=Ylc7QbOi1WY)

${ }^{18}$ Tales como Memoria Chilena (www.memoriachilena.cl) y el Museo de la Memoria y Derechos Humanos (http://ww3.museodelamemoria.cl/)

\section{Referencias bibliográficas}

Ancán, J. (2014). De küme molfüñche a 'civilizados a medias': liderazgos étnicos e intelectuales mapuche en la Araucanía fronteriza (1883-1930). Polis, Revista de la Universidad Bolivariana, 13(38), 19-44.

Brown, D. \& Nichols, G. (2012). Protecting indigenous cultural property in the age of digital democracy: Institutional and communal responses to Canadian First Nations and Maori heritage concerns. Journal of Material Culture, 17(3), 307-324.

Christakis, N. \& Fowler, J. (2011). Connected. How your friends' friends' friends affect everything you feel, think and do. New York: Back Bay Books.

Gajjala, R. (2013). Cyberculture and the Subaltern. Weavings of the Virtual and Real. Lanham: Lexington Books.

Golumbia, D. (2014). Death of a Discipline. Differences: A Journal of Feminist Cultural Studies, 25(1), 156-176. doi: 10.1215/104073912420033

Janicke, S., Efer, T., Büchler, M. \& Scheuermann, G. (2015). Designing Close and Distant Reading Visualizations for Text Re-use. En Computer Vision, Imaging and Computer Graphics - Theory and Applications. (pp. 153-171). Suiza: Springer International Publishing.

Jimeno, M. (Ed). (2014). Juan Gregorio Palechor: The Story of My Life. Durham, NC: Duke UP.

Menard, A. (2013). Libro Diario del Presidente de la Federación Araucana. Santiago: CoLibris.
${ }^{19}$ En la entrevista "Critical Intimacy: An Interview with Gayatri Chakravorty Spivak", 29 de julio, 2016, dice "I spend my life trying to make people understand that we should claim how useful we are and not just give in to the definitions of how to make ourselves useful by complete digitizing and all that stuff...If you don't train the soul, the global/digital cannot be used right."

Moretti, F. (2000). Conjectures on World Literature. New Left Review, 1, 54-69. Recuperado de: https://newleftreview.org/ll/1/ franco-moretti-conjectures-on-world-literature

(2011). Network Theory and Plot Analysis. New Left Review, 68. Recuperado de: https://newleftreview.org/ll/68/francomoretti-network-theory-plot-analysis

Pavez, J. (2003). Mapuche ñi nütram chilkatun / Escribir la historia mapuche. Revista de Historia Indígena, 7, 7-53. Recuperado de: http://www.revistahistoriaindigena.uchile.cl/index.php/RHI/article/ viewFile/40157/41720

Paulson, S. (2016). Entrevista Critical Intimacy: An Interview with Gayatri Chakravorty Spivak. Recuperado de: https://lareviewofbooks. org/article/critical-intimacy-interview-gayatri-chakravorty-spivak

Spiro, L. (2012). This Is Why We Fight. Defining the Values of the Digital Humanities. En Debates in the Digital Humanities (pp. 16-35). Minneapolis: Universidad de Minnesota Press. Recuperado de http://dhdebates.gc.cuny.edu/debates/text/13

Spivak, G. (2016). Seminario sobre violencia, étnicidad y género. Seminario Internacional: Los velos de la violencia: Reflexiones y experiencias étnicas y de género en Chile y América Latina. Simposio llevado a cabo en el Centro Interdisciplinario de Estudios de Género, Santiago, Chile. Digital Humanities Quarterly, Alliance of Digital Humanities Organizations, 4(1). Recuperado de: http:// digitalhumanities.org/dhq/vol/4/1/000080/000080.html 